\title{
Feasibility of satellite image and GIS sampling for population representative surveys: a case study from rural Guatemala
}

\author{
Ann C. Miller ${ }^{1 *+} \mathbb{0}$, Peter Rohloff ${ }^{1,2,3 \dagger}$, Alexandre Blake ${ }^{4}$, Eloin Dhaenens ${ }^{3}$, Leah Shaw ${ }^{3}$, Eva Tuiz ${ }^{3}$, \\ Francesco Grandesso ${ }^{4}$, Carlos Mendoza Montano ${ }^{5}$ and Dana R. Thomson ${ }^{6}$
}

\begin{abstract}
Background: Population-representative household survey methods require up-to-date sampling frames and sample designs that minimize time and cost of fieldwork especially in low- and middle-income countries. Traditional methods such as multi-stage cluster sampling, random-walk, or spatial sampling can be cumbersome, costly or inaccurate, leading to well-known biases. However, a new tool, Epicentre's Geo-Sampler program, allows simple random sampling of structures, which can eliminate some of these biases. We describe the study design process, experiences and lessons learned using Geo-Sampler for selection of a population representative sample for a kidney disease survey in two sites in Guatemala.

Results: We successfully used Epicentre's Geo-sampler tool to sample 650 structures in two semi-urban Guatemalan communities. Overall, $82 \%$ of sampled structures were residential and could be approached for recruitment. Sample selection could be conducted by one person after 30 min of training. The process from sample selection to creating field maps took approximately $40 \mathrm{~h}$.

Conclusion: In combination with our design protocols, the Epicentre Geo-Sampler tool provided a feasible, rapid and lower-cost alternative to select a representative population sample for a prevalence survey in our semi-urban Guatemalan setting. The tool may work less well in settings with heavy arboreal cover or densely populated urban settings with multiple living units per structure. Similarly, while the method is an efficient step forward for including non-traditional living arrangements (people residing permanently or temporarily in businesses, religious institutions or other structures), it does not account for some of the most marginalized and vulnerable people in a populationthe unhoused, street dwellers or people living in vehicles.
\end{abstract}

Keywords: Population-representative study, Sampling frame, Guatemala, Simple random sample, Sample selection

\section{Background}

Population-representative household survey methods require up-to-date, accurate sampling frames and sample designs that minimize time and cost of fieldwork. These requirements are particularly important in low- and

\footnotetext{
*Correspondence: Ann_miller@hms.harvard.edu

${ }^{\dagger}$ Ann C. Miller and Peter Rohloff contributed equally to this manuscript

1 Department of Global Health and Social Medicine, Harvard Medical School, 641 Huntington Ave, Boston, MA, USA

Full list of author information is available at the end of the article
}

middle-income countries (LMICs) where other sources of data, such as census data and civil registration data are expensive to maintain, and likely to be out of date or incomplete [1]. Routine national surveys including the Demographic Health Surveys (DHS) and Multiple Indicator Cluster Surveys (MICS) uniquely generate data needed for program and policy planning, monitoring development goals, and tracking development progress. However, they take place approximately every 5 years in most settings and may be out of date at time points of

c) The Author(s) 2020. This article is licensed under a Creative Commons Attribution 4.0 International License, which permits use, sharing, adaptation, distribution and reproduction in any medium or format, as long as you give appropriate credit to the original author(s) and the source, provide a link to the Creative Commons licence, and indicate if changes were made. The images or other third party material in this article are included in the article's Creative Commons licence, unless indicated otherwise in a credit line to the material. If material is not included in the article's Creative Commons licence and your intended use is not permitted by statutory regulation or exceeds the permitted use, you will need to obtain permission directly from the copyright holder. To view a copy of this licence, visit http://creativeco mmons.org/licenses/by/4.0/. The Creative Commons Public Domain Dedication waiver (http://creativecommons.org/publicdomain/ zero/1.0/) applies to the data made available in this article, unless otherwise stated in a credit line to the data. 
interest. Due to weak national data systems, household surveys are also often the main source of disease prevalence estimates in LMICs.

In 2017, while planning a household survey in two areas of Guatemala to estimate prevalence of chronic kidney disease of unknown etiology $(\mathrm{CKDu})$, we considered available tools and methods for selecting a populationrepresentative sample of households. At the time, the 2018 Guatemala Census was being planned, and only 2002 Census data were available. We therefore considered several additional options.

\section{Multi-stage cluster sampling}

In multi-stage cluster samples, small areas are first selected at random from municipal or governmental maps, usually based on the last census, with probability proportionate to population size. The implied assumption is that the relative proportion of population in each small area-or "cluster"-has not changed substantially since the last census. In countries with extremely outdated census data, modelled gridded population estimates have instead been used to sample clusters using free (e.g. GridSample.org) or hired bespoke (e.g. RTI International's Geo-Sampling) gridded population sampling tools [2]. A complete enumeration of all households is then conducted in the selected clusters using foot travel and hand-drawn sketch mapping. Finally, households are randomly selected from within the enumerated clusters for inclusion in the study. While multi-stage cluster samples are widely considered to optimize statistical efficiency and fieldwork effort, sampling from either the available 2002 Guatemala census, or a gridded population dataset derived from this outdated census, were considered poor options (Table 1).

\section{Multi-stage sampling with random-walk initiation}

Other traditional methods, including the World Health Organization's former Expanded Programme on Immunization (EPI) survey design, use two stage cluster samples [3]. In the EPI design, the first stage clusters are selected with probability proportional to population size from census enumeration areas (government estimates); however, individual households are selected through a "spin-the-pen" and random walk mechanism. In this method, a pen or bottle is placed at a central location of the cluster and spun. Households in the indicated direction are identified, and one is selected at random to be the first household of the sample. The rest are selected in relation to that household in either a "next-nearest" fashion, or a skip pattern, in which, for example, every third

Table 1 Pros and cons of sampling approaches and tools considered

\begin{tabular}{|c|c|c|}
\hline Approach & Pros & Cons \\
\hline \multicolumn{3}{|l|}{ Multi-stage cluster sampling } \\
\hline Census frame with manual cluster selection & $\begin{array}{l}\text { Calculate weights and Cls } \\
\text { Low cost of } 1 \text { st-stage cluster selection } \\
\text { Does not require skills beyond survey statistics }\end{array}$ & $\begin{array}{l}\text { Outdated 1st-stage frame } \\
\text { High cost of } 2 \text { nd-stage household enumeration }\end{array}$ \\
\hline Gridded population frame with GridSample.org & $\begin{array}{l}\text { Calculate weights and Cls } \\
\text { Low cost of 1st-stage cluster selection } \\
\text { Requires few skills beyond survey statistics }\end{array}$ & $\begin{array}{l}\text { Outdated 1st-stage frame } \\
\text { High cost of } 2 \text { nd-stage household enumeration }\end{array}$ \\
\hline Gridded population frame with RTI Geo-Sampling & $\begin{array}{l}\text { Calculate weights and Cls } \\
\text { Requires few skills beyond survey statistics }\end{array}$ & $\begin{array}{l}\text { Outdated 1st-stage frame } \\
\text { High cost of } 1 \text { st-stage cluster selection } \\
\text { High cost of } 2 \text { nd-stage household enumeration }\end{array}$ \\
\hline Census frame with EPI design & $\begin{array}{l}\text { Low cost of } 1 \text { st-stage cluster selection } \\
\text { Low cost of } 2 \text { nd-stage } \\
\text { Does not require skills beyond survey statistics }\end{array}$ & $\begin{array}{l}\text { Outdated 1st-stage frame } \\
\text { Cannot calculate weights and } \mathrm{Cls}\end{array}$ \\
\hline \multicolumn{3}{|l|}{ Spatial sampling } \\
\hline No stratification & & $\begin{array}{l}\text { Sample not representative of the population } \\
\text { Requires GIS skills }\end{array}$ \\
\hline Stratification on structure density & $\begin{array}{l}\text { Updated frame } \\
\text { Calculate weights and Cls }\end{array}$ & $\begin{array}{l}\text { Requires GIS and other skills beyond survey } \\
\text { statistics } \\
\text { High cost of structure enumeration (or similar } \\
\text { count of buildings) }\end{array}$ \\
\hline \multicolumn{3}{|l|}{ Simple random sample of structures } \\
\hline $\begin{array}{l}\text { Enumerate all structures in OpenStreetMap, } \\
\text { Google Earth, or GIS software }\end{array}$ & $\begin{array}{l}\text { Updated frame } \\
\text { Calculate weights and Cls }\end{array}$ & $\begin{array}{l}\text { High cost of structure enumeration } \\
\text { Requires GIS skills }\end{array}$ \\
\hline Epicentre Geo-Sampler & $\begin{array}{l}\text { Updated frame } \\
\text { Calculate weights and Cls } \\
\text { Low cost of structure selection (no enumeration) } \\
\text { Requires few skills beyond survey statistics }\end{array}$ & \\
\hline
\end{tabular}


or fifth house in the direction is sampled. This method, while commonly used for decades, can introduce bias $[4,5]$, as households nearest the centrally located start point will be more likely to be selected than households at a settlement's periphery, and assumptions about nonresponse must be made; not every household has the same (or a known) probability of selection. This method was not suitable because it would require use of an outdated census simple frame and would not permit calculation of sample weights needed to produce unbiased estimates and confidence intervals of disease prevalence (Table 1).

\section{Spatial samples}

Other surveys have attempted to employ spatial sampling approaches, for example in setting up a cholera vaccination campaign in Democratic Republic of Congo [7], a basic pediatric health indicators survey in Zambia [8], or a diabetes prevalence survey in Guatemala [9]. Often, these samples are constructed through generation of a regular geometric grid, from which points are selected through simple, systematic, stratified or clustered random sampling for inclusion in the study. Spatial samples have also been used to map risk to human health across space (e.g. pollution [10] or distribution of species [11]), and were combined with methods such as capture-recapture and adjustment for population density $[11,12]$.

Importantly, simple spatial samples do not result in population-representative samples because human populations are not distributed uniformly across geographic areas. Simple spatial sampling designs lead to oversampling of sparsely distributed households. In rural areas, this means that remote households will be more likely sampled than in settlements, and in urban areas, wealthier households, which often have larger areal footprints, will be more likely sampled than small, densely packed poor households, resulting in biased population samples and biased results. Stratification by population density prior to random spatial sampling has been used in population surveys [10], requiring population proportional to size sampling and weighting to account for that in analysis (Table 1).

\section{Simple random sample of enumerated households}

The most statistically efficient survey design would be a simple random sample of households from the entire population if an updated, complete household sample frame were available. Surveys with smaller coverage areas have selected simple random samples of households, for example in China, using electronic listings of households in two districts [6], and Democratic Republic of Congo [13] using civil registry data for household listings of small catchment areas. However, resource-limited countries like Guatemala rarely have a unique home address system or database of household GPS locations. Alternatively, satellite data can be employed to digitize all structures within an area [14], though this is time and labor intensive, and requires geographic skills and knowledge that are not always available in resource-poor settings (Table 1). However, in humanitarian emergencies, online volunteer communities can be of assistance with digitization and providing some of these digitization skills for researchers in LMICs.

Epicentre, a Non-Governmental Organization working with Doctors Without Borders, has recently created a new sampling tool, known as Geo-Sampler, to assist researchers in generating household samples using satellite imagery, eliminating the need for cluster-level sampling. Geo-Sampler does this via a Google Earth-based interface by generating random points within a polygon (e.g. district, or city) superimposed on up-to-date satellite imagery. Users can optionally set a radius around the point (e.g. 10 meters to cover the size of a typical building), and incrementally select an infinite number of random points. Importantly, in the Geo-Sampler protocol, all points that do not include a structure are discarded, resulting in a sample of structures rather than geographic units, which overcomes the limitations of simple random spatial sampling (illustrated in Fig. 1). Additional data about structure occupancy and population density are collected during the survey and used to generate sample weights that adjust for non-populated structures or structures with multiple households (Table 2).

To generate a population-representative sample from an up-to-date sample frame with maximum statistical power and efficient field protocols, we used Geo-Sampler with protocols adapted to our setting and study needs. Although some studies led by Epicentre have used earlier versions of Geo-Sampler to select households within a two-stage cluster design $[15,16]$, as yet, no articles detail its use as a tool to generate a full sample. Therefore, here we describe our experience with this new tool in designing and conducting a population survey for estimating the prevalence of chronic kidney disease in two areas of Guatemala; Tecpán, Department of Chimaltenango (core population, excluding outlying settlements approximately 84,000) and San Antonio Suchitepéquez, Department of Suchitepéquez (core population approximately 52,000).

\section{Results}

\section{Sample selection}

Of the main sample of 350 structures, 288 (82\%) were residential, 81 (23\%) had more than one building per residence, and 17 (5\%) had more than one family per structure (Table 2). This was different between the two sites. Tecpán reported statistically significantly more 


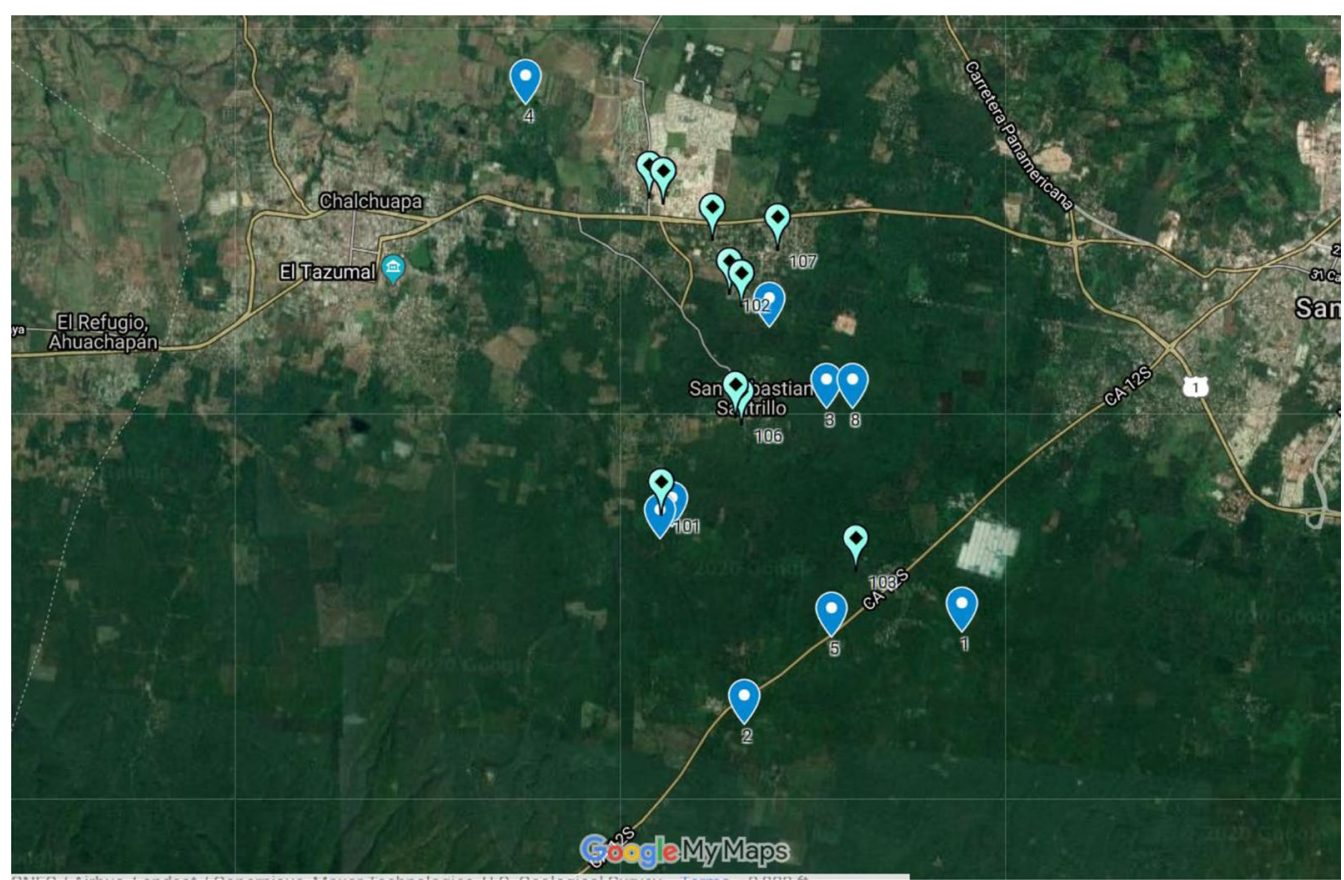

Fig. 1 Example of Comparison of Simple Spatial Sampling (dark blue markers 1-10) vs. Simple random sample of structures (light blue markers 101-110) in which only those selected points that contain a structure were retained. Both were generated by Geo-Sampler

Table 2 Results of approaches to households in CKD prevalence survey, Guatemala

\begin{tabular}{|c|c|c|c|c|}
\hline Variable & Tecpán N (\%) & Suchitepéquez N (\%) & Total N & $P$ value \\
\hline Structure sample size & 220 & 130 & 350 & 0.04 \\
\hline Residences & $174(79.1)$ & $114(87.7)$ & 288 & \\
\hline Non residences/vacant & $46(20.1)$ & $16(12.3)$ & 62 & \\
\hline Median [IQR] structures per residence & $1[1-5]$ & $1[1-3]$ & & \\
\hline $\begin{array}{l}N(\%) \text { with more than one household per structure } \\
(n=158)\end{array}$ & $7(9.0)$ & $10(12.2)$ & & \\
\hline Household responses & 174 & 114 & 288 & 0.008 \\
\hline Recruited & $110(63.2)$ & $78(68.4)$ & 188 & \\
\hline Refused & $57(32.7)$ & $23(20.2)$ & 80 & \\
\hline Ineligible or uncontactable & $7(4.0)$ & $13(11.4)$ & 20 & \\
\hline
\end{tabular}

non-residential buildings $(44,20.0 \%)$ than San Antonio Suchitepéquez $(15,11.5 \%)$ in the main sample, and significantly more structures composing a residence (mean of 1.84 in Tecpán vs. 1.40 in San Antonio Suchitepéquez, $\mathrm{p}<0.01$ ). No differences were seen between the two sites with respect to the number of multi-household structures.

\section{Feasibility}

We found sample selection using Epicentre's Geo-Sampler tool to be feasible and practicable in this setting (see Table 3 for efficiences and challenges). Sample selection was conducted by one person (ACM), after receiving approximately $30 \mathrm{~min}$ of training on the Geo-Sampler tool from a contact at Epicentre $(\mathrm{AB})$. Creating the sample dataset in our study required three steps (described in detail in the "Methods" section): selecting the point-withbuffer sample, recording sampled structure coordinates, and creating field maps, although updates to Geo-Sampler now allow the structure coordinates to be created and downloaded to a spreadsheet within the tool. The process of selecting the 650 main and replacement coordinates took approximately $24 \mathrm{~h}$. The process of manually digitizing and recording sampled structure coordinates 
Table 3 Efficiencies and challenges of using Geo-Sampler tool and protocols for population-level data collection in Guatemala

\begin{tabular}{|c|c|c|}
\hline Steps in sampling protocol & Efficiencies & Challenges and considerations for future work \\
\hline 1. Training of study staff on Geo-Sampler Tool & $\begin{array}{l}\text { Professional contacts facilitated access to Epicen- } \\
\text { tre staff }\end{array}$ & $\begin{array}{l}\text { Currently, limited technical documentation } \\
\text { When available, technical documentation is in } \\
\text { limited languages } \\
\text { Formal training on the tool is not currently available }\end{array}$ \\
\hline $\begin{array}{l}\text { 2. Selecting samples and digitizing sampled } \\
\text { structure coordinates }\end{array}$ & $\begin{array}{l}\text { No special software required } \\
\text { Geo-Sampler tool using very up-to-date, high } \\
\text { resolution imagery, so we were able to identify } \\
\text { recently constructed structures } \\
\text { Sample can be selected at one time, by one } \\
\text { person } \\
\text { Sample of structures behind walls can be easily } \\
\text { selected } \\
\text { Geo-Sampler does not retain the list of selected } \\
\text { structure coordinates after the program is shut } \\
\text { down, which limits privacy concerns }\end{array}$ & $\begin{array}{l}\text { Sample selection required significant expert time } \\
\text { If updated samples are needed, extra time from } \\
\text { someone trained in the tool will be required, } \\
\text { unless multiple people are trained } \\
\text { If multiple people are trained and are selecting the } \\
\text { sample, significant coordination and oversight } \\
\text { would be required to ensure quality control, con- } \\
\text { sistency, and to eliminate repetition of structures, } \\
\text { unless adaptations are made to Geo-Sampler to } \\
\text { allow for simultaneous use of multiple users }\end{array}$ \\
\hline 3. Locating selected structures in the field & $\begin{array}{l}\text { Use of the Android/Google Maps platform was } \\
\text { intuitive and well-known to local study staff, } \\
\text { cost-saving } \\
\text { Satellite overlay on Google Maps useful not } \\
\text { only for finding tagged structures but also } \\
\text { identifying principal entrances, alleyways, etc. } \\
\text { when attempting to approach structures (many } \\
\text { located in walled compounds, etc.) } \\
\text { Selecting structures rather than relying upon } \\
\text { investigators' concepts of what a"residence" } \\
\text { looked like allowed us to include non-traditional } \\
\text { living situations }\end{array}$ & $\begin{array}{l}\text { Initial version of the Geo-Sampler tool provided. } \\
\text { kml files but without identifiable latitude and lon- } \\
\text { gitude, which then required a } 2 \text { stage process to } \\
\text { determine. This was changed during the course of } \\
\text { the study by Epicentre } \\
\text { Saved efficiencies of multi-stage sampling some- } \\
\text { what offset by inefficiency of inevitably many } \\
\text { tagged structures not being residences. } \\
\text { This method does not allow for people who do not } \\
\text { live in structures. People living on the street or in } \\
\text { cars would still be left out of these surveys } \\
\text { A few coordinates selected were close enough that } \\
2 \text { different structures were given the same study } \\
\text { ID by different data collectors. This was discov- } \\
\text { ered and addressed in the data cleaning phase by } \\
\text { recoding one of the residences of each pair } \\
\text { Connectivity in our sites in Guatemala was gener- } \\
\text { ally good, but we experienced frequent signal } \\
\text { drop-outs, requiring large-format printed physical } \\
\text { map back-up at all times. This would likely be the } \\
\text { case in many LMIC settings, especially rural areas. } \\
\text { Drop-outs in connectivity also caused rapid } \\
\text { phone battery drain (due to searching signal), } \\
\text { and required staff to carry multiple recharging } \\
\text { packets when in the field in order to keep phones } \\
\text { charged }\end{array}$ \\
\hline
\end{tabular}

took approximately another $12.5 \mathrm{~h}$. The process of creating field maps took about two hours, for a total of $38.5 \mathrm{~h}$. These skills required for this work were limited, and can be performed by anyone with basic spreadsheet skills, familiarity with Google Earth, an initial training in the Geo-Sampler tool, and sufficient familiarity with geographic coordinate systems.

The data collection field team initially consisted of two community nurses with licensed practical nurse (LPN)equivalent degrees and one supervisor with masters-level in nursing. While this level of training was necessary for our study to collect biologic samples and conduct interviews, advanced degrees are not necessary to use GeoSampler outputs to identify households. Only familiarity with smartphone technology, map literacy, and Google
Maps is required. A 2 day training and pilot exercise was sufficient to allow the data collectors to get started. Time between map generation and first data collection in the field was 2 days.

Data cleaning was conducted throughout the study by two people at a time, one of the coinvestigators (ACM) and the project coordinator (serially, ED LS, ET) with support from the PI (PR). All data cleaners and analysts had a Masters or PhD. Skill required for data cleaning include familiarity with excel, Redcap and Stata programming. Calculation of sample weights after data cleaning took approximately 3 days. Calculation of sample weights involved sophisticated demography skills for devising the formulae, and strong MS excel skills to program the spreadsheets. Application of the sampling 
and response weights to generate prevalence estimates required advanced Stata programming skills, but once the programs were created, they could be reused swiftly. Investigators on the team (ACM, DRT, PR) have strong excel skills, statistical programming software skills (Stata 15), and/or advanced population health and demography skills.

\section{Discussion}

We have identified several advantages of this method over the more traditional methods of sampling frame enumeration in this area of Guatemala. The main advantages are those of time, cost and statistical efficiency.

The traditional enumeration method of household census or sending a mapping crew ahead of time to knock on doors is time-consuming and expensive. DHS suggests estimating 2 months for this phase in their field manuals [17], and another 2 months between household enumeration and data collection. In a smaller survey of 1600 households in rural Madagascar, enumeration required 20 days with 9 field teams, and another 24 days between enumeration and the initiation of data collection [18]. Our survey required a total of 1 week's hours (38.5) of full-time work (across six non-consecutive weeks) by one person to generate the maps plus 1 week of training and 2 days from beginning of sample selection to beginning of field time. In this area of Guatemala, many households are located behind privacy or security walls and residents may not admit enumeration crew to their homes, leaving the crew to simply guess at the number of households behind the walls. This area of Guatemala is not heavily forested, so satellite imagery gives an excellent representation of the structures available for sampling. Additionally, Geo-Sampler's use of high-resolution satellite imagery captured within 6 months of the initiation of the field work provided a much more up-to-date source of data than typically used to select cluster surveys.

Furthermore, surveys which digitized all structures from satellite imagery as a sample frame required intimate knowledge of the area including typical structures and living patterns to be able to produce a reasonable map of residential buildings in the area. The digitization process is quite time-consuming and relies on the twostep process of first enumeration followed by selection. The Geo-Sampler method does not require digitizing all structures, and allows for simultaneous enumeration and selection, at a very rapid pace. Because each point in the polygon has an equal chance of being selected, and the user has the ability to select only points with a structure contained within the buffer as part of the sample, statistical correction for clustering is not required. In this lower-resource context, the absence of both (1) a recent national census and the corresponding updated sampling frame and (2) an updated accurate gridded population dataset, Geo-Sampler allowed us to select a simple random sample of households from a random sample of structures, rather than a multi-stage clustered sample, which also enabled us to reduce our sample size and conduct the study with a reduced budget.

For our study, Geo-Sampler had certain advantages over some other available options. First, it provided us with a simple, user-operated sample selection, and unlike proprietary tools such as RTI's Geosampling tool [17], Epicentre's Geo-Sampler was free. Another advantage to Geo-Sampler is that the tool is preloaded with Google satellite imagery. Although there are other free options to select a simple random sample of structures, most rely on Volunteered Geographic Information (VGI) from OpenStreetMap which is generally derived from older and coarser Bing satellite images [18]. While the open access of these map databases is appealing and useful, debate about the data quality and level of bias inherent in these crowdsourced datasets continues [19, 20]. VGI mapping programs have historically been shown to have greater reporting and detail in urban areas with representation skewed toward high-income countries [21]. Some form of expert gate-keeping has been suggested as both a remedy for these possible inaccuracies, and a remedy for the perception of inaccuracies, which leads to mistrust of these free and open datasources [19]. However, as these free software programs continue to expand and grow, with heightened attention to measuring data quality $[18,22]$ the tradeoff between VGI and other systems will become less important.

Cost, however, can be a major barrier to the ability of researchers in LMICs to conduct their own population-level surveys, especially in the absence of grant funding or partnerships with universities. This may reinforce existing research inequities between low and high resourced settings. Our survey included the use of several proprietary licensed programs that were available through universities, including the Microsoft packages and ArcGIS. Lower or no-cost programs exist that could be valid substitutes when resources are scarce, and if researchers do not have university partnerships to draw upon. However, tradeoffs may still need to be considered between cost and quality of tools, especially when considering open, crowdsourced programs.

This method does have some possible limitations when implemented. The areas of Guatemala in which the study was conducted vary greatly in terms of environment, climate, and wealth. However, they are similar in their levels of arboreal cover and that most of the buildings are single family houses. Although a small proportion of the population in each site reside in apartment buildings or other multi-family dwellings, the vast majority of families 
reside in single-family houses. These tools and protocols are well-suited to this scenario, but may be less applicable to densely populated urban settings with multiple living units per structure. Despite the overall successful implementation of the protocols, our study team did face a few challenges in the use of the protocols during the field work. The study coordinator used a mobile device to identify a selected structure, visited it first to determine if it was actually a household, and if so, enrolled any eligible participants. The study nurses then followed up at a pre-arranged time to conduct study activities. On a few occasions, the randomly selected coordinates were close enough that two different structures were given the same study ID by different data collectors. This was discovered during the cleaning process and one of the households was reassigned to a different number. However, this was a time-consuming puzzle. Connectivity was also an issue; the data collectors experienced frequent signal drop-outs, and used a lot of battery life in searching for connectivity. This issue will probably be applicable to other lower-resource settings as well, so use of navigation applications that do not require internet connectivity are recommended. We addressed this by providing large-format printed maps as a back-up. Harnessing the knowledge locally hired staff have of their neighborhoods is also important, and including names of neighborhoods was useful to support the ability of nurses to easily find houses. Although data collectors had extensive experience with smart phones, some study staff were less comfortable using both paper maps and the phone-based Google Maps. Despite these challenges, nurse data collectors have been successfully able to locate and identify the selected households for inclusion into the study, replacing with others as necessary.

Although these methods provide a step forward in including non-traditional living arrangements (people residing permanently or temporarily in businesses, religious institutions or other structures), they do not capture some of the most marginalized and vulnerable people in the population-the unhoused, street dwellers or people living in vehicles. Some of the structures selected were in areas determined to be too dangerous to the safety of data collectors to enroll. Thus, there remains a group of community residents whose health indicators, perspectives and risks are invisible and undocumented. Further research on ways to include these most vulnerable groups in population studies is needed.

\section{Conclusion}

In combination with our design protocols, the Epicentre Geo-Sampler tool provided a feasible, rapid and lowercost alternative to select a representative population sample for a prevalence survey in our setting.

\section{Methods \\ Description of the main study and study area}

This research is part of an National Institutes of Health R21-funded study (1R21TW010831-01) on chronic kidney disease of unknown origin $(\mathrm{CKDu})$, which emerged as a recent epidemic in Central America and other global sites in individuals without traditional CKD risk profiles (young, male, without diabetes or hypertension) [24]. The main objective of the study is to estimate the prevalence of both CKD and CKDu in two communities with different risk profiles. The study uses the infrastructure of the healthcare organization Wuqu' Kawoq|Maya Health Alliance which has a long-standing presence in both study communities. Tecpán, Department of Chimaltenango (core population, excluding outlying settlements, is approximately 84,000 ) is a majority indigenous Maya community in the temperate highlands of central Guatemala (elevation 7500 feet). San Antonio Suchitepéquez, Department of Suchitepéquez (core population is approximately 52,000) is a lowland Pacific coastal warm-climate town with larger non-indigenous population (elevation 1000-1500 feet). The sites were chosen to provide a diversity of CKD risk factors, including differential exposure to heat stress and strenuous agricultural labor, profiles of pesticides in common use, diet diversity, risk for obesity and cardiovascular disease, and early life insults such as child malnutrition.

The prevalence survey was designed to be conducted through home visits by study nurses, consisting of an interview with any eligible household members (nonpregnant adults who agree to participate and provide informed consent), biometric measurements using a bioimpedance scale, urine samples to measure urine protein and creatinine, and serum samples to measure serum creatinine and glycosylated hemoglobin $\mathrm{A} 1 \mathrm{C}$. These test procedures allow for screening for diabetes and CKD and permit staging of any diagnosed CKD using Kidney Disease: Improving Global Outcomes(KDIGO) guidelines [25]. Positive results are returned to the participants by the study team along with confirmatory testing and a facilitated referral to public health or specialty clinics, as needed, for any follow-up care. 


\section{Constructing the sampling frame and random sampling of structures and households}

An a priori sample size calculation of 700 people from 350 households was required to estimate the point prevalence of CKD with a margin of error of $0.35-220$ households from Tecpán and 130 from San Antonio Suchitepéquez-including inflation for refusals, household clustering in structures, and an expected prevalence of $10 \%$ [26].

Using ArcGIS 10.15.1, we created polygon shapefiles for Tecpán and San Antonio Suchitepéquez. Shapefiles were drawn to include the municipal boundaries of each town, with some expansion at the edges to include households when town boundaries cut through a tight group of structures. These maps were reviewed and approved by the co-investigators with knowledge of the area (PR, $\mathrm{CM}$ ). We then imported the shapefiles into Geo-Sampler.

The Geo-Sampler tool (version 0.1.0.47 (2018-05-02)) interfaces with Google Earth and randomly selects points within a specified polygon shape, following a sampling with replacement technique. We set a buffer of $15 \mathrm{~m}$ around each sampling point, approximately the size of a lot in these regions of Guatemala, to reduce the number of points that needed to be selected and potentially dropped. Sample selection was performed beginning in April 2018 for Tecpán and May 2018 for San Antonio Suchitepéquez, and the imagery for both study sites in Google Earth was dated January 9, 2018.

One of the co-authors (ACM) selected and reviewed each point (with $15 \mathrm{~m}$ buffer) according to the following rules:

- If a structure existed within the buffer, the point was kept, and the structure within the buffer was included in the sample.

- If more than one structure existed within the buffer and the centroid point fell between them, the structure with any wall or corner nearest to the centroid point (based on visual estimation) was selected.

- If the closest structures were equidistant to the point (based on visual estimation), we selected the structure closest to 12:00, considering all structures in a clockwise fashion from 12:00.

- All sample structures were kept, regardless if they appeared to be non-residential, multi-family, or part of compounds (e.g., outdoor kitchen or privy).

- Additional points with $15 \mathrm{~m}$ buffers were selected until the target sample size was reached.

By using a buffer around points, points did not always fall directly over a structure. To record sampled structure locations, we used Google Earth (same 1/9/2018 imagery) to manually digitize latitude and longitude coordinates for the sampled structures, and recorded these in a .xls spreadsheet file. Given potential structure abandonment and non-residential structures, an extra 155 replacement structures were selected in Tecpán and 145 replacement clusters were selected in San Antonio Suchitepéquez. At the end of each Geo-Sampling session, a .csv file of retained sample points, and .xls file of corresponding sampled structures' latitude and longitude coordinates were saved. Because sampling with replacement was used, each selected structure was compared with its nearest neighbors to be sure that the same structure was not included more than once.

Hard copy maps of each structure with a Google Earth base layer at a finer resolution were also provided to the data collectors for navigation.

\section{Approaching household and calculating sampling weights} The protocol for approaching households was as follows: the data collectors were given a list of selected structures with coordinates and maps from the main sample $(\mathrm{n}=220$ in Tecpán, and $\mathrm{n}=130$ in Suchitepéquez) and separate lists of coordinates and maps of replacement structures. If the structure was a residence, the data collectors initiated enrollment activities for a household. If the structure was not part of a residence, the data collectors attempted to identify the nearest residence, turning in a clockwise circle on the street in front of the structure. If none of the structures in that circle were residences (or if there were no other structures), the data collectors made a note and a structure from the replacement list was visited instead. If the structure was a residence that contained more than one household, a list of the households was made and one household was selected at random. For each household, queries outlined in Table 4 were collected in order to appropriately weight the survey results.

Sample weights were calculated as follows, using stratum (city), structure and household response rates. Notation below uses $k$ for strata, $j$ for structures and $i$ for households.

$$
w_{i j k}=\frac{D_{i j k}}{d_{i j k}} \times T_{j i k} \times \frac{G_{k} / g_{k}}{M_{k}} \times \frac{m_{k}}{m_{k *}} .
$$

where: $\mathrm{w}_{\mathrm{ijk}}$ is the household weight. $\mathrm{G}_{\mathrm{k}}$ is the total population in stratum $\mathrm{k}$ (using estimated projections by the Guatemala National Institute of Statistics for the municipalities of Tecpán and San Antonio Suchitepéquez [27]). $\mathrm{g}_{\mathrm{k}}$ is the average household size in stratum $\mathrm{k}$ as estimated by Guatemala DHS 2014-2015 [28]. $\mathrm{M}_{\mathrm{k}}$ is the number of target households in stratum $k . \mathrm{D}_{\mathrm{ijk}}$ is the number of households $i$ enumerated in structure $j$ in stratum $k \cdot \mathrm{d}_{\mathrm{ijk}}$ is the number of households $i$ selected in structure $j$ in 
Table 4 Data collected on each sampled structure

\begin{tabular}{|c|c|}
\hline Query & Possible Responses \\
\hline Is the selected structure a residence? ${ }^{a}$ & Yes/No \\
\hline How many associated structures are in use by the household? & Number of structures and type ${ }^{b}$ \\
\hline Does more than one household live in structure? & Number of households in residence \\
\hline Recruitment outcomes & $\begin{array}{l}\text { Number of households approached } \\
\text { Number of households with a contact } \\
\text { Number of households with at least one member } \\
\text { recruited ("hh enrolled") } \\
\text { Number of eligible adults in household and whether } \\
\text { each individual was recruited or declined participa- } \\
\text { tion }\end{array}$ \\
\hline
\end{tabular}

a Including structures of multiple use (stores, churches, etc.) as long as also a residence

b For example, selected structure might be the primary residence for the household, but there may also be a separate kitchen structure and garage structure for the same household

stratum $k . \mathrm{T}_{\mathrm{jik}}$ is the number of structures $j$ in household $i$ in stratum $k \cdot \mathrm{m}_{\mathrm{k}}$ is the number of approached households in stratum $k$ during fieldwork. $\mathrm{m}_{\mathrm{k}^{*}}$ is the number of responded households in stratum $k$ during fieldwork.

\section{Pilot exercise}

Prior to the initiation of formal data collection, a pilot was conducted with study data collectors to determine whether use of hand-held devices using readily available commercial GPS software (Google Maps) was feasible for data collectors following a brief training to identify households using latitude and longitude coordinates. Other methods were also tested, including the use of a Garmin GPS device and printed copies of maps. The training took one afternoon and consisted of didactics and examples. The pilot consisted of field work with the 2 data collectors and the study coordinator, who were given 10 structure coordinates to identify, and printed maps with the structures marked. Using their office-issued mobile Android phones (models Motorola MotoE4 Plus, Samsung Galaxy J3, Samsung A10) the nurses programmed the coordinates into the phones and used the factory-installed directional navigation software to find the structures, with realtime field assistance and as-needed support from one of the study investigators (PR). In this pilot, the study staff were able to program coordinates into the phones and easily find assigned structures, and so we elected to proceed with hand-held devices with Google Maps rather than other methods. This was cost-saving, as it was not necessary to purchase GPS devices. All staff were also already familiar with the use of Google Maps.

\section{Abbreviations}

CKD: Chronic Kidney disease; CKDu: Chronic Kidney Disease of unknown etiology; DHS: Demographic and Health Surveys; EPI: Expanded Programme on
Immunization; GPS: Geographic positioning system; KDIGO: Kidney Disease: Improving Global Outcomes; LMIC: Low- and middle-income countries; LPN: Licensed practical nurse; MICS: Multiple Indicator Cluster Surveys; NIH: National Institutes of Health.

\section{Acknowledgements}

The authors gratefully acknowledge the hard work of the data collection teams, specifically Irma Yolanda Raquec Teleguario, Glenda Gómez Hernández, and Mérida Isabel Coj Sajvin, Héctor Gomez Hernández, Alvaro Danilo Sir López and staff at Wuqu'Kawok. We acknowledge the support of the INCAP laboratories, and Dr. Joaquin Barnoya for productive conversations. We gratefully acknowledge the work of Serge Balandine from Epicentre on design of Geo-Sampler and Epicentre for their cooperation in allowing us to use this tool. We especially thank the study participants, without whom this would not be possible

\section{Authors' contributions}

PR Principal Investigator of the CKDu study; designed study, contributed to data acquisition, co-wrote first and all drafts of the manuscript. ACM designed study, conducted sample selection, all data analysis and interpretation, wrote first and all drafts of manuscript. AB helped test the Geo-Sampler tool, interpreted results, contributed to multiple drafts of manuscript, reviewed and approved final draft. ED assisted in design of field study, contributed to data collection and cleaning, reviewed and approved final draft of manuscript. LS contributed to data collection and cleaning, contributed to multiple drafts of manuscript, reviewed and approved final draft. ET led final data cleaning, contributed to analysis, reviewed and approved final draft of manuscript. FG:was involved in design and application of Geo-Sampler tool, and reviewed the final draft of the manuscript. CMM Co-PI of the CKDu study, assisted with interpretation of results, reviewed and approved final draft of manuscript. DRT contributed to design of study, designed analysis and mentored ACM on conduct of analysis, contributed to interpretation of results, co-wrote first and all drafts of manuscript. All authors read and approved the final manuscript.

\section{Funding}

This study was funded via the National Institutes of Health R21 grant FIC R21 TW010831-02: Building capacity for chronic kidney disease research in Guatemala (Peter Rohloff, PI; Carlos Mendoza co-PI).

\section{Availability of data and materials}

Datasets created for this manuscript are not publically available because they are coordinates of specific structures and residences of study participants; these data will not be made public for confidentiality reasons. However, a deidentified replication dataset from the entire CKDu study will be made available at the Principal Investigator's Harvard University's Dataverse following publication of final study results. 


\section{Ethics approval and consent to participate}

:The study was reviewed and approved by the Partners Health Care, Maya Health Alliance, and the Instituto de Nutricion de Centro América y Panama's respective Institutional Review Board.

\section{Consent for publication}

All authors have reviewed the manuscript and agree to its publication.

\section{Competing interests}

Alexandre Blake worked for Epicentre on the Geo-Sampler tool between 2015 and 2018. Francesco Grandesso works for Epicentre. The authors declare no other competing interests.

\section{Author details}

${ }^{1}$ Department of Global Health and Social Medicine, Harvard Medical School, 641 Huntington Ave, Boston, MA, USA. ${ }^{2}$ Division of Global Health Equity, Brigham and Women's Hospital, Boston, MA, USA. ${ }^{3}$ Wuqu' Kawoq, Maya Health Alliance, Santiago Sacatepéquez, Guatemala. ${ }^{4}$ Epicentre, Paris, France. ${ }^{5}$ Institute of Nutrition of Central America and Panama (Instituto de Nutrición de Centroamérica, INCAP), y Panamá, Guatemala City, Guatemala. ${ }^{6}$ Department of Social Statistics and Demography, University of Southampton, Southampton, England.

Received: 13 August 2020 Accepted: 19 November 2020

Published online: 05 December 2020

\section{References}

1. AbouZahr C, de Savigny D, Mikkelsen L, Setel PW, Lozano R, Lopez AD. Towards universal civil registration and vital statistics systems: the time is now. Lancet. 2015;386(10001):1407-18.

2. Cajka J, Amer S, Ridenhour J, Allpress J. Geo-sampling in developing nations. Int J Social Res Methodol. 2018;21(6):729-46.

3. Immunization EPo. The EPI coverage survey: Traning for midlevel managers. In: World Health Organization Dol, Vaccines and Biologicals, editor. WHO/IVB/0807. Geneva, Swizterland: WHO Press; 2008.

4. Lemeshow S, Tserkovnyi AG, Tulloch JL, Dowd JE, Lwanga SK, Keja J. A computer simulation of the EPI survey strategy. Int J Epidemiol. 1985;14(3):473-81.

5. Milligan P, Njie A, Bennett S. Comparison of two cluster sampling methods for health surveys in developing countries. Int J Epidemiol. 2004;33(3):469-76.

6. Lv J, Liu QM, Ren YJ, Wang WF, Gong T, Li LM. Application of a simple random sampling method on surveys at the community level. Zhonghua Liu Xing Bing Xue Za Zhi. 2010:31(4):421-3.

7. Massing LA, Aboubakar S, Blake A, Page AL, Cohuet S, Ngandwe A, et al. Highly targeted cholera vaccination campaigns in urban setting are feasible: The experience in Kalemie, Democratic Republic of Congo. PLoS Negl Trop Dis. 2018;12(5):e0006369.

8. Lowther SA, Curriero FC, Shields T, Ahmed S, Monze M, Moss WJ. Feasibility of satellite image-based sampling for a health survey among urban townships of Lusaka Zambia. Trop Med Int Health. 2009;14(1):70-8.

9. Bream KDW, Breyre A, Garcia K, Calgua E, Chuc JM, Taylor L. Diabetes prevalence in rural Indigenous Guatemala: A geographic-randomized cross-sectional analysis of risk. PLOS ONE. 2018;13(8):e0200434.

10. Kumar N. Spatial sampling design for a demographic and health survey. Popul Res Pol Rev. 2007;26:581-99.

11. Cole Burton A, Neilson E, Moreira D, Ladle A, Steenwag R, Fisher JT, et al. Wildlife camera trapping: A review and recommendations for linking surveys to ecological processes. J Appl Ecol. 2015;52:675-85.

12. Sollmann R, Gardner B, Belant J. How does spatial study design influence density estimates from spatial capture-recapture models? PLoS ONE. 2012;7(4).
13. Maketa V, Mavoko HM, da Luz RI, Zanga J, Lubiba J, Kalonji A, et al. The relationship between Plasmodium infection, anaemia and nutritional status in asymptomatic children aged under five years living in stable transmission zones in Kinshasa Democratic Republic of Congo. Malar J. 2015;14:83.

14. Wagenaar BH, Augusto O, Asbjornsdottir K, Akullian A, Manaca N, Chale $F$, et al. Developing a representative community health survey sampling frame using open-source remote satellite imagery in Mozambique. Int J Health Geogr. 2018;17(1):37.

15. Grandesso F, Allan M, Jean-Simon PS, Boncy J, Blake A, Pierre R, et al. Risk factors for cholera transmission in Haiti during inter-peak periods: insights to improve current control strategies from two case-control studies. Epidemiol Infect. 2014;142(8):1625-35.

16. Luquero FJ, Grout L, Ciglenecki I, Sakoba K, Traore B, Heile M, et al. First outbreak response using an oral cholera vaccine in Africa: vaccine coverage, acceptability and surveillance of adverse events, Guinea, 2012. PLoS Negl Trop Dis. 2013;7(10):e2465.

17. Survey Organization Manual for Demographic and Health Surveys. Calverton. MD: ICF International; 2012.

18. Enquete de Base: District De Ifanadiana, Madagascar. Antananarivo, Madagascar: Institut National des Statsitiques, Madagascar (INSTAT) PIVOT, Madagascar Ministere de la Sante Publique, Madagascar; 2014 August, 2014

19. Basiri A, Haklay M, Foody G, Mooney P. Crowdsourced geospatial data quality: challenges and future directions. Int J Geographical Inform Sci. 2019;33(8):1588-93.

20. Basiri A, Jackson M, Amirian P, Pourabdollah A, Sester M, Winstanley A, et al. Quality assessment of OpenStreetMap data using trajectory mining. Geo-spatial Inform Sci. 2016;19(1):56-68.

21. Ma D, Sandberg M, Jiang B. Characterizing the heterogeneity of the OpenStreetMap data and community. Int J Geo-Inform. 2015;4:535-50.

22. Senaratne H, Mobasheri A, Loai Ali A, Capineri C, Haklay M. A review of volunteered geographic information quality assessment methods. Int J Geographical Inform Sci. 2016;31(1):139-67.

23. Costa Fonte C, Antoniou V, Bastin L, Estima J, Arsanjani JJ, Laso Bayas J-C, et al. Assessing VGI Data Quality. In: Foody G, See L, Fritz S, Mooney P, olteanu-Raimond A-M, Costa Fonte C, et al., editors. Mapping and the Citizen Sensor: ubiquity Press; 2017.

24. Hoy W, Ordunez P. Epidemic of Chronic Kidney Disease in Agricultural Communities in Central America. Case definitions, methodological basis and approaches for public health surveillance. Washington, D.C.: Pan American Health Organization; 2017.

25. KDIGO. KDIGO 2012 Clinical Practice Guidelinefor the Evaluation and Management of Chronic Kidney Disease. Kidney International Supplements; 2013

26. Hill NR, Fatoba ST, Oke JL, Hirst JA, O'Callaghan CA, Lasserson DS, et al. Global prevalence of chronic kidney disease - a systematic review and meta-analysis. PLoS ONE. 2016;11(7):e0158765.

27. Estadistica INd. Guatemala: Estimaciones de la Población total por municipio. Período 2008-2020. 2020 http://www.oj.gob.gt/estadisticaj/ reportes/poblacion-total-por-municipio(1).pdf.

28. Encuesta Nacional de Salud Materno Infantil 2014-2015. Informe Final.: Ministerio de Salud Pública y Asistencia Social (MSPAS) Instituto Nacional de Estadística (INE) ICF International; 2017.

\section{Publisher's Note}

Springer Nature remains neutral with regard to jurisdictional claims in published maps and institutional affiliations. 